\title{
Multifractal patterns formed by laser irradiation in GeAl thin multilayer films
}

\author{
Angel Sánchez \\ Departamento de Física Teórica I, Universidad Complutense, Ciudad Universitaria, E-28040 Madrid, Spain \\ Rosalía Serna, Fernando Catalina, and Carmen N. Afonso \\ Instituto de Optica, Consejo Superior de Investigaciones Científicas, Serrano 121, E-28006 Madrid, Spain
}

(Received 30 January 1992)

\begin{abstract}
Structures induced in GeAl thin multilayer films by laser irradiation are studied. We compute their multifractal spectra from digitized transmission-electron micrographs. The results show that the patterns, which arise from a diffusion process followed by rapid solidification, are fractal, and that they cannot be described by a unique scaling exponent.
\end{abstract}

\section{INTRODUCTION}

Fractal $^{1,2}$ growth processes have been the subject of extensive experimental, numerical and theoretical studies in the last decade. ${ }^{3,4}$ Processes that give rise to the formation of very complex structures include, for example, dendritic solidification in an undercooled medium, viscous fingering, or electrochemical deposition. Fractal dimensions ${ }^{1-4}$ have been widely employed in an attempt to relate the geometry of these structures to corresponding growth mechanisms. However, it has been recently suggested that multifractal scaling ${ }^{5}$ can be necessary to account for these and many other patterns appearing in nature. Thus, the growth probability distribution of diffusion-limited aggregation ${ }^{6}$ (DLA) clusters exhibit multifractal properties, ${ }^{7}$ and examples of this multifractal character of the growth probabilities have already been found experimentally in ammonium chloride crystals (Ref. 8) and iron oxide (Ref. 9) and Co (Ref. 10) magnetic particle aggregates. The geometry (see Sec. III below; see also Ref. 4 for a precise definition) of DLA clusters has also been found to have multifractal character ${ }^{11}$ however, it has been claimed that the up-to-date studied physical examples of DLA are not multifractal ${ }^{12}$ in this geometrical sense.

Germanium compounds have previously received much attention as prototypes of the complicated behaviors happening in nonequilibrium solidification and/or crystallization of this and other materials. ${ }^{4,13}$ Thus, treeshaped fractal crystals have been grown by heat treatment of amorphous $\mathrm{GeSe}_{2}$ thin films, ${ }^{14}$ and DLA-like clusters, whose shape and dimension depends on the $\mathrm{Au}$ grain size appear upon ion implantation and annealing of GeAu bilayers. ${ }^{15,16}$ Besides, crystallization of amorphous $\mathrm{Al}_{x} \mathrm{Ge}_{1-x}$ thin films annealed at around $500 \mathrm{~K},{ }^{17,18}$ or spontaneous crystallization in laser-induced amorphous areas on $\mathrm{Al}_{0.6} \mathrm{Ge}_{0.4}$ thin films ${ }^{19}$ show the so-called densebranching morphology, ${ }^{17}$ which is a nonfractal although very intricated kind of pattern.

In this work we aim to provide evidence for geomet- rically multifractal patterns formed in GeAl thin multilayer films by laser irradiation. The process we report in this paper, which has been thoroughly described elsewhere ${ }^{20}$ takes place in a range of temperatures and solidification velocities different from those of the above mentioned researches. In Sec. II we describe both the experimental and numerical procedures, including a brief account of previous work ${ }^{20}$ regarding our samples. Section III contains the main part of the paper, including a detailed description of our computations and a tentative discussion of possible physical reasons for the appearance of multifractal patterns. Section IV concludes the paper summarizing our results.

\section{EXPERIMENTAL AND NUMERICAL PROCEDURE}

We work with four-layered thin films, with alternate layers of $\mathrm{Ge}$ and $\mathrm{Al}$, a 50 at. \% Al composition, a total film thickness in the $60-\mathrm{nm}$ range, and a $\mathrm{Ge}$ layer at the surface. They are irradiated in air by means of single nanosecond pulses from a $\mathrm{Xe}-\mathrm{Cl}$ excimer laser with energy densities in the range $20-70$ $\mathrm{mJ} \mathrm{cm}{ }^{-2}$. Using Rutherford-backscattering spectrometry and transmission-electron microscopy (TEM) it is found ${ }^{20}$ that the as-grown films consist of amorphous $\mathrm{Ge}$ and crystalline Al layers, and that laser irradiation induces the mixing of the layers; moreover, the degree of such mixing increases as laser energy density increases. This process occurs by diffusion of the elements within the liquid state followed by a rapid solidification process, which involves cooling rates in the $10^{9} \mathrm{~K} \mathrm{~s}^{-1}$ range.

Among all the microstructures arising from laser irradiation, ${ }^{20}$ we are interested in the Al cellular crystals formed at medium $\left(\simeq 45 \mathrm{~mJ} \mathrm{~cm}^{-2}\right)$ energy densities. Typical TEM micrographs of these samples are shown in Fig. 1: Al crystals are seen there as dark regions, the background being an amorphous GeAl mixture. The degree of darkness of each crystal depends essentially on the concentration of $\mathrm{Ge}$ that has diffused into them. We 

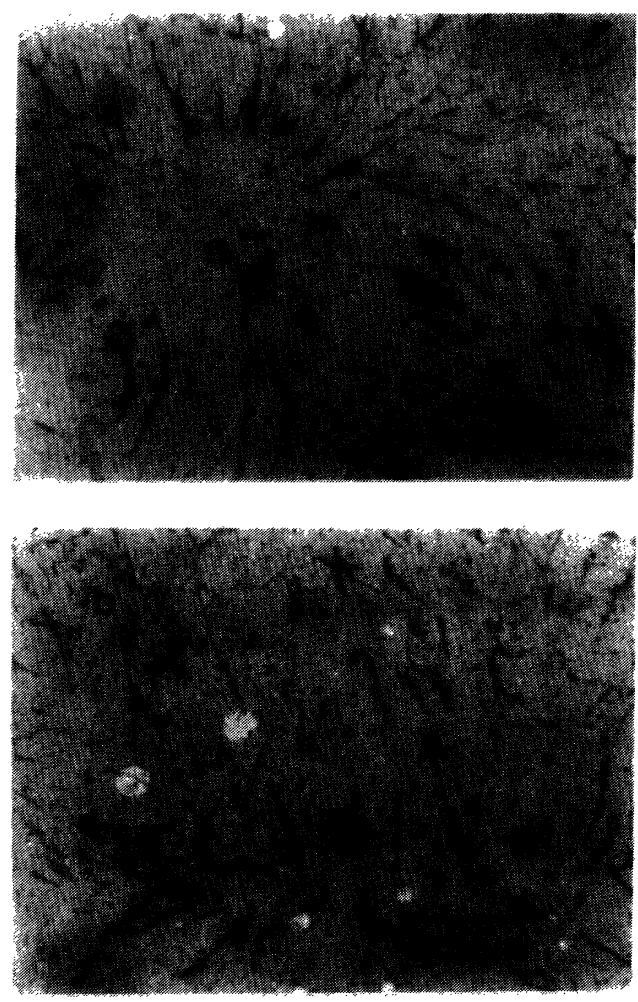

FIG. 1. TEM images of two areas, (a) and (b), of GeAl four-layered films after laser irradiation at $45 \mathrm{~mJ} \mathrm{~cm}^{-2}$.

want to point out that these crystals form a quasi-twodimensional pattern because their thickness is at most (definitely much less than) the film thickness $(60 \mathrm{~nm})$, while their typical length is around $500 \mathrm{~nm}$; so, we can ignore the fact that TEM micrographs are actually twodimensional projections of a three-dimensional structure.

Different micrographs including $\mathrm{Al}$ crystals in an amorphous matrix were digitized, measuring the optical density at $512 \times 512$ pixels, and their generalized dimensions and multifractal spectrum ${ }^{2,4,5}$ were computed. To be precise, the values provided by the digitizer are modified according the following rule: they are set to zero if they do not belong to a crystal (i.e., their optical density is less than or equal to that of the background) and they are taken to be the difference between their optical density and that of the background if they do belong to a crystal (i.e., their optical density is greater than the background value). This defines a positive measure on the set of crystals, which we finally normalize dividing each value among the sum of all of them. We have obtained the multifractal spectrum by two different methods. The first and simplest one was usual box-counting (see, e.g., Refs. 2, 4, 5, and 8) scaling (range, 1-64 pixels) of the sums of the $q$ th power of the measure contained in each box; this yields directly the generalized dimensions $D_{q}$ and subsequently the multifractal spectrum $f(\alpha)$ by a numerical Legendre transform. As is well known that this procedure suffers from enormous uncertainties in the negative $q$ region, coming from finite size and resolution effects among other factors, we have checked our results with a second, more accurate technique proposed

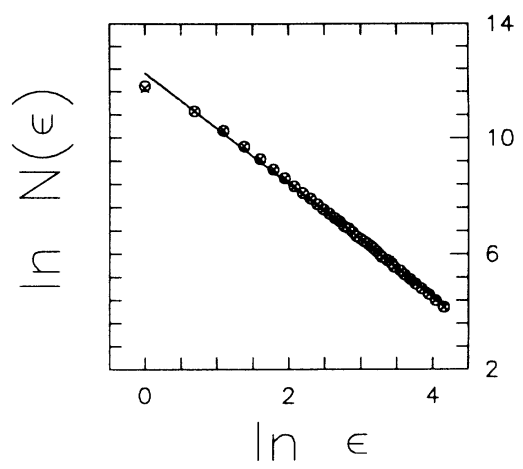

FIG. 2. Log-log plot to compute the box-counting dimension $D_{0}$ for micrographs 1(a) (empty circles) and 1(b) (crosses). The line is the least-squares fit, with slopes $1.88 \pm 0.09$ and $1.89 \pm 0.08$, respectively.

by Chhabra and Jensen. ${ }^{21}$ Their algorithm gives directly the multifractal spectrum $f(\alpha)$ by pure scaling without invoking further numerical treatment and has very useful properties. Its description is a bit long, and hence we refer the reader to Ref. 21 .

\section{RESULTS AND DISCUSSION}

Our results, shown in Figs. 2-4, indicate clearly that the set of Al crystals, with the measure we have introduced above, has a well-defined multifractal structure. As an example of the scaling range and the quality of the corresponding power-law behavior, we show in Fig. 2 the log-log graph from which the box-counting $\left(D_{0}\right)$ dimension is obtained; other dimensions $D_{q}$ are obtained by means of analogously good fits, especially for positive $q$. As for $D_{0}$, it turns out to be $1.88 \pm 0.09$, where we also take into account the slight shifts induced by the different backgrounds. Several remarks are in order regarding our computations. First, the spectrum is rather insensitive either to the precise value of the background threshold, a property that we have checked, computing the spectra taking different background values in a wide range, or to the specific area of the sample whose spectrum we compute, as we have also verified by studying several of them. Second, there is an extremely good agreement be-

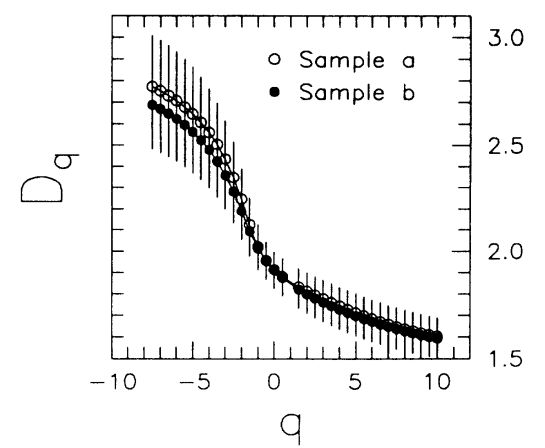

FIG. 3. Generalized dimensions $D_{q}$ for micrographs 1(a) (empty circles) and 1(b) (full circles). Line is only a guide to the eye. 


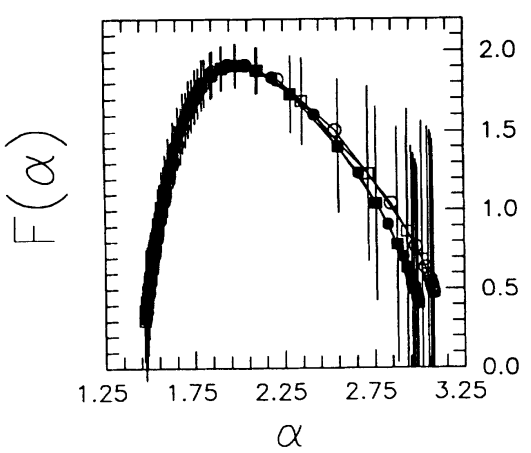

FIG. 4. Multifractal spectra $f(\alpha)$ for micrographs 1(a) (empty circles and squares) and 1(b) (full circles and squares). Line is only a guide to the eye. Circles are computed by numerical Legendre transform of the generalized dimensions shown in Fig. 3, squares are obtained from Chhabra-Jensen algorithm.

tween both methods for computing the $f(\alpha)$ spectrum, which can be seen in Fig. 4. Third, even with the improved Chhabra-Jensen algorithm, the computation error is large in the negative $q$ region; however, these errors are (at least in a $q$ interval near zero) not compatible with a single dot for $f(\alpha)$, which would correspond to a usual fractal (nonmultifractal), ${ }^{2,4}$ a result strongly supported by the precise calculation for positive $q$. Finally, we have checked the verification of known mathematical properties, with good results: it can be seen from Figs. 3 and 4 that $f\left(\alpha=D_{1}\right)=D_{1}$, and that the straight line with unit slope is tangent to $f(\alpha)$ at that point, as should happen. ${ }^{2,4}$

We have also carried out some computations on the crystals' geometry, suppressing the distinction between regions with different degrees of darkness by setting all nonzero pixels to a value 1 (equivalently, now the measure is zero if the considered pixel does not belong to an $\mathrm{Al}$ crystal and one if it does belong to an $\mathrm{Al}$ crystal). By this procedure we may distinguish between multifractality coming from the fact that we have a nonuniform measure defined on a single fractal, or geometric multifractality 4,11 induced by the very structure of the pattern. Unfortunately, this two-valued measure gives rise to much larger errors, and we have only been able to obtain reliable values for the generalized dimensions for positive $q$, which we plot in Fig. 5. Our results are not conclusive as to whether the geometry of crystals is multifractal or not, but it is clear that its multifractal nature is much less pronounced than that arising when the optical density is taken into account.

Concerning the question of the physical reasons for the formation of these multifractal patterns, we believe that the main factor influencing the process is the very rapid solidification through which those structures are produced. Indeed, there exists a close relationship between cooling rates and pattern formation: low solidification velocities $\left(\sim 10^{3}-10^{6} \mathrm{~K} \mathrm{~s}^{-1}\right)$ usually lead to dendritic growth and crystalline metastable phases, whereas higher velocities $\left(\sim 10^{6}-10^{8} \mathrm{~K} \mathrm{~s}^{-1}\right)$ give rise to $\alpha$ - $\mathrm{Al}$ plus $\mathrm{GeAl}$ amorphous phases. ${ }^{19,22}$ In our case, the process is even faster $\left(10^{9} \mathrm{~K} \mathrm{~s}^{-1}\right)$, and it happens in two

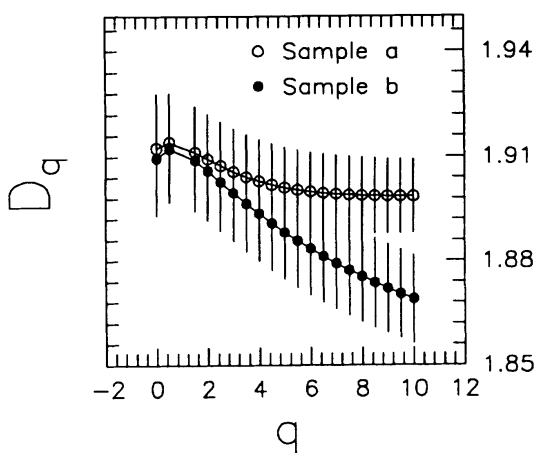

FIG. 5. Generalized dimensions when all nonzero pixels have been set to one for micrographs 1(a) (empty circles) and 1(b) (full circles). Line is only a guide to the eye.

stages: first, the laser pulse melts the multilayer film which immediately begins to mix through liquid phase diffusion; second, when energy deposition ends, the diffusion front is suddenly quenched. As the structure of diffusion fronts behaves similarly to those of percolation processes (see, e.g., Ref. 23), it could be possible that we are seeing crystals grown at clusters that were originated by a percolationlike phenomenon. Crystals would grow only in these clusters because their $\mathrm{Al}$ content would be high enough to become nucleant for crystallization but not too large as to prevent $\mathrm{Ge}$ from diffusing inside the $\mathrm{Al}$ crystals (see below). As an estimation, we may recall that the $\mathrm{Al}$ percolation threshold for $\mathrm{GeAl}$ systems is rather high, around 0.6 (see, Ref. 18 and references therein): hence, only the deep, Al-rich region near the substrate would support those $\mathrm{Al}$ clusters. It is also important to realize that if the diffusion process were completed, the sample would become homogeneous, and $\mathrm{Al}$ cellular crystals could not be formed. Finally, Ge diffuses into the $\mathrm{Al}$ clusters, enhances their contrast in the micrographs and seems to be mainly responsible for the multifractal measure. This factor imposes a further spatial limit for the process, namely, that it must happen near the innermost layer interface reached by the liquid front in order to have some Ge diffused into liquid $\mathrm{Al}$ but in an amount small enough to inhibit amorphous phase formation; this conclusion is in agreement with the location of the $\mathrm{Al}$ crystals seen in the irradiated samples. ${ }^{20}$ The whole process would then occur in a thin slice of the sample near the substrate. The underlying percolation class phenomenon might also explain the large value $(1.88 \pm 0.09)$ of the fractal dimension of the patterns (recall that two-dimensional percolation clusters near the threshold have similar dimensions ${ }^{2,4}$ ).

\section{CONCLUSION}

Laser irradiation can induce formation of multifractal patterns in GeAl thin multilayer films. Their generalized dimensions and multifractal spectra computed by two methods are in very good agreement. A possible explanation for the physical origin of such multifractality is suggested, based on the fact that a diffusion process can form percolationlike interface structures through a rapid solidification process, preventing the appearance of other patterns like dendrites, which in addition are formed at 
much lower quenching rates. Nevertheless, further theoretical work is needed to completely explain this phenomenon.

\section{ACKNOWLEDGMENTS}

We acknowledge useful conversations with F. Guinea, as well as his critical reading of the manuscript. We want to thank M. Pérez for assistance with the microdensitometer and J. A. Cuesta for help with the computer code. One of us (A.S.) is grateful to L. Vázquez for encouragement. This work was partially supported by the Comisión Interministerial de Ciencia y Tecnología (CICyT) of Spain under Project Nos. MAT88-0437 and MAT90-0544.
${ }^{1}$ B. B. Mandelbrot, The Fractal Geometry of Nature (Freeman, San Francisco, 1982).

${ }^{2}$ J. Feder, Fractals (Plenum, New York, 1988).

${ }^{3}$ On Growth and Form: Fractal and Non-Fractal Patterns in Physics, edited by H. E. Stanley and N. Ostrowsky (Martinus Nijhoff, Dordrecht, 1983); Fractals in Physics, edited by L. Pietronero and E. Tosatti (North-Holland, Amsterdam, 1986); Random Fluctuations and Pattern Growth, edited by H. E. Stanley and N. Ostrowsky (Kluwer Academic, Dordrecht, 1988).

${ }^{4}$ T. Vicsek, Fractal Growth Phenomena (World Scientific, Singapore, 1989).

${ }^{5}$ B. B. Mandelbrot, J. Fluid Mech. 62, 331 (1974); H. G. E. Hentschel and I. Procaccia, Physica 8D, 435 (1983); U. Frisch and G. Parisi, in Turbulence and Predictability in Geophysical Fluid Dynamics and Climate Dynamics, edited by M. Ghil, R. Benzi, and G. Parisi (North-Holland, Amsterdam, 1985); T. C. Halsey, M. H. Jensen, L. P. Kadanoff, I. Procaccia, and B. Shraiman, Phys. Rev. A 33, 1411 (1986).

${ }^{6}$ T. A. Witten and L. M. Sander, Phys. Rev. Lett. 47, 1400 (1981).

${ }^{7}$ Y. Hayakawa, S. Sato, and M. Matsushita, Phys. Rev. A 36, 1963 (1987).

${ }^{8}$ S. Ohta and H. Honjo, Phys. Rev. Lett. 60, 611 (1988).

${ }^{9}$ L. J. Huang, B. X. Liu, J. R. Ding, and H. H. Li, Phys. Rev. B 40, 858 (1989).

${ }^{10}$ J. R. Ding and B. X. Liu, Phys. Rev. B 40, 5834 (1989).

${ }^{11}$ T. Vicsek, F. Family, and P. Meakin, Europhys. Lett. 12, 217 (1990).

${ }^{12}$ F. Argoul, A. Arneodo, G. Grasseau, and H. Swinney, Phys.
Rev. Lett. 61, 2558 (1988); F. Argoul, A. Arneodo, J. Elezgaray, G. Grasseau, and R. Murenzi, Phys. Rev. A 41, 5537 (1990).

${ }^{13}$ H. Jones, Mater. Sci. Eng. 65, 145 (1984).

${ }^{14}$ G. Radnoczy, T. Vicsek, L. M. Sander, and D. Grier, Phys. Rev. A 35, 4012 (1987).

${ }^{15}$ Hou Jian-Guo, Wu Zi-Qin, and Bi Ling-Song, Thin Solid Films 173, 77 (1989).

${ }^{16}$ Hou Jian-Guo and Wu Zi-Qin, Phys. Rev. B 42, 3271 (1990).

${ }^{17}$ E. Ben-Jacob, G. Deutscher, P. Garik, N. D. Goldenfeld, and Y. Lereah, Phys. Rev. Lett. 57, 1903 (1986); G. Deutscher and Y. Lereah, Phys. Rev. Lett. 60, 1510 (1988); S. Alexander, R. Bruinsma, R. Hilfer, G. Deutscher, and Y. Lereah, Phys. Rev. Lett. 60, 1514 (1988).

${ }^{18}$ F. Catalina, C. N. Afonso, and C. Ortiz, Thin Solid Films 167, 57 (1988).

${ }^{19}$ C. N. Afonso, F. Catalina, and J. Solís, Mater. Sci. Eng. B 7, 169 (1990).

${ }^{20}$ C. N. Afonso, R. Serna, F. Catalina, N. Teixeira, M. F. da Silva, and J. C. Soares, Appl. Phys. A 52, 69 (1991).

${ }^{21}$ A. B. Chhabra and R. V. Jensen, Phys. Rev. Lett. 62, 1327 (1989).

${ }^{22}$ C. N. Afonso, J. Solís, and F. Catalina, Appl. Surf. Sci. 43, 171 (1989).

${ }^{23}$ B. Sapoval, M. Rosso, and J.-F. Gouyet, J. Phys. (Paris) Lett. 46, L419 (1985); M. Rosso, J.-F. Gouyet, and B. Sapoval, Phys. Rev. B 32, 6053 (1985); Phys. Rev. Lett. 57, 3195 (1986); V. B. Sapozhnikov and M. G. Goldiner, J. Phys. A 23, 5309 (1990). 

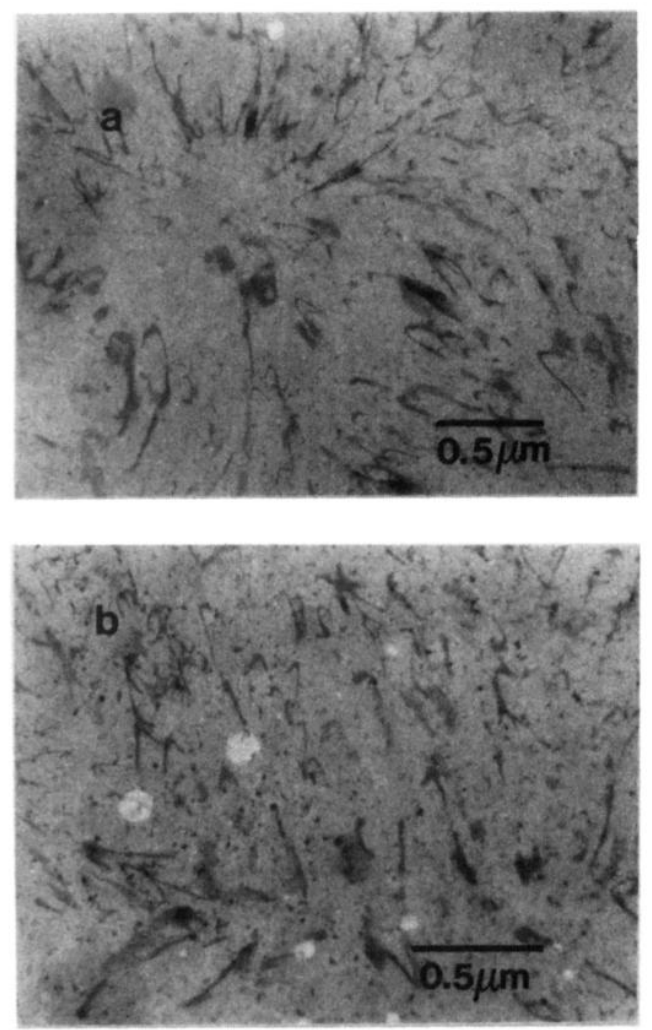

FIG. 1. TEM images of two areas, (a) and (b), of GeAl four-layered films after laser irradiation at $45 \mathrm{~mJ} \mathrm{~cm}^{-2}$. 